\title{
Zentralvorstandssitzung vom 16. Mai 2013
}

\begin{abstract}
Versorgungsforschung ISPM Bern - Die FMH hat von 2010 bis 2012 das ISPM Bern mit einem Sponsoring des Forschungsschwerpunkts Versorgungsforschung unterstützt. Das Thema ist nach wie vor wichtig, und die regelmässigen Informationen sowie der Austausch in der Dialoggruppe haben sich bewährt. Deshalb entscheidet der ZV, das Sponsoring für drei weitere Jahre (2014-2016) fortzuführen.
\end{abstract}

Schweizerisches Tarifsystem Psychiatrie (TARPSY) Für die stationäre Psychiatrie soll ein schweizweit einheitliches, leistungsorientiertes Tarifsystem (TARPSY) erarbeitet werden. Auf dessen Gestaltung kann die FMH sowohl über ihre beiden Beobachter im Steuerungsausschuss TARPSY als auch über das Koordinationsgremium und den Verwaltungsrat der SwissDRG AG Einfluss nehmen. Der ZV genehmigt das von der FMH-Begleitgruppe TARPSY verfasste Positionspapier.

SwissDRG-Version 3.0 - Die Neuerungen der SwissDRG-Version 3.0 wirken sich unterschiedlich aus. Die Entwicklung zu mehr Zusatzentgelten und die bessere Abbildung von hochaufwendigen Fällen sind zwar positiv, aber zum Beispiel die ungenügende Datenqualität und die erheblichen Schwankungen der Kostengewichte sind zu bemängeln. Der $\mathrm{ZV}$ unterstützt daher die Stellungnahme, welche das Ressort erstellt hat.
Arzneimittelinformationssystem (AIPS) - Das Arzneimittelinformationssystem (AIPS) von Swissmedic, welches seit Anfang Jahr das Kompendium abgelöst hat, genügt den Anforderungen der Ärzteschaft nicht. Viele Ärztinnen und Ärzte wissen nicht, wo sie die früher im Kompendium abgedruckten Informationen beziehen können. An einem von der FMH einberufenen runden Tisch sollen alle Beteiligten eine gemeinsame Nachfolgelösung finden.

Epidemiengesetz - Der ZV beschliesst, dass die FMH das Abstimmungskomitee für die Revision des Epidemiengesetzes unterstützen soll. Weitere Anfragen bei anderen Organisationen des Gesundheitswesens sind noch hängig. Zudem soll der FMH-Präsident Einsitz im Abstimmungskomitee nehmen.

BAG-Strategie zur Masernelimination - Das BAG hat eine Kommunikationsstrategie zur Masernelimination erarbeitet. Ziel ist es, künftig einen Durchimpfungswert von über $95 \%$ bei Kindern zu erreichen und bis 2015 die unter 50-Jährigen nachzuimpfen. Der ZV entscheidet, die Kommunikationskampagne von 2013 bis 2015 ideell zu unterstützen. Ausserdem wird die Leiterin des Ressorts Gesundheitsförderung und Prävention im Maserneliminationskomitee mitwirken.

Aktuelle Forumthemen
Diskutieren Sie mit! Im Forum präsentieren wir regel-
mässig brisante Themen aus Politik, Ökonomie
und Wissenschaft, die das Schweizer Gesundheitswesen
betreffen. Bringen Sie Ihre Meinung ein oder kom-
mentieren Sie die Äusserungen Ihrer Kolleginnen und
Kollegen. Das Forum finden Sie unter:
www.saez.ch/forum/

